\title{
Das ZEWO-Gütesiegel für gemeinnützige NRO als Beitrag zu erhöhter Transparenz auf dem Schweizer Spendenmarkt
}

\section{Ariuscha Davatz}

\section{(2) OpenEdition}

\section{Journals}

Electronic version

URL: http://journals.openedition.org/sjep/501

DOI: $10.4000 /$ sjep.501

ISSN: 1663-9677

\section{Publisher}

Institut de hautes études internationales et du développement

\section{Printed version}

Date of publication: 1 novembre 2004

Number of pages: 117-124

ISBN: 2-88247-057-6

ISSN: $1660-5926$

\section{Electronic reference}

Ariuscha Davatz, « Das ZEWO-Gütesiegel für gemeinnützige NRO als Beitrag zu erhöhter Transparenz auf dem Schweizer Spendenmarkt », Schweizerisches Jahrbuch für Entwicklungspolitik [Online], 23-2 | 2004, Online erschienen am: 10 Juni 2010, abgerufen am 08 September 2020. URL : http:// journals.openedition.org/sjep/501; DOI : https://doi.org/10.4000/sjep.501 


\title{
Das ZEWO-Gütesiegel für gemeinnützige NRO als Beitrag zu erhöhter Transparenz auf dem Schweizer Spendenmarkt
}

\author{
Ariuscha Davatz*
}

\section{Geschichte der ZEWO}

Bereits im Jahr 1934 wünschten Spenderinnen und Spender in der Schweiz über die Vertrauenswürdigkeit gemeinnütziger Organisationen Bescheid zu wissen, damit sie ihr Geld gut einsetzen konnten. Aufgrund dieses Bedürfnisses gründeten die Landeskonferenz für soziale Arbeit (LAKO) und die Schweizerische Gemeinnützige Gesellschaft 1934 die „Zentralauskunftsstelle“ und 1936 den Verein ZEWO (Zentralstelle für Wohlfahrtsunternehmen). 1942 wurde das Gütesiegel für gemeinnützige, Spenden sammelnde Organisationen mit den entsprechenden Richtlinien geschaffen. Organisationen, welche das Gütesiegel erhielten, waren bereit, über die gesetzlichen Mindestanforderungen hinaus zusätzliche Standards zu erfüllen. Die ZEWO führte von Beginn an einen Auskunftsdienst für die Spenderinnen und Spender und organisiert seit 1946 den Schweizer Sammlungskalender.

Am 20. Juni 2001 beschloss die Mitgliederversammlung, den Verein in eine Stiftung umzuwandeln, um die Unabhängigkeit von den Hilfswerken zu erhöhen.

\section{Die ZEWO heute}

Ziel der Stiftung ZEWO ist es, die Transparenz und Lauterkeit gemeinnütziger, Spenden sammelnder Organisationen gegenüber der Öffentlichkeit zu fördern. Dazu vergibt sie ein Gütesiegel an gemeinnützige Organisationen und sorgt für dessen Bekanntheitsgrad. Das Gütesiegel wird jeweils für fünf Jahre verliehen. Nach Ablauf dieser Zeit werden die Organisationen mit der Rezertifizierung erneut eingehend auf die Einhaltung der ZEWO-Anforderungen geprüft. Als Dienstleistung für die gemeinnützigen Organisationen mit dem ZEWO-Gütesiegel koordiniert die ZEWO die Sammlungen der grössten Hilfswerke im Schweizer Sammlungskalender. Für die Spenderinnen und Spender führt die ZEWO einen Informations- und Auskunftsdienst zu Organisationen mit und ohne Gütesiegel. Zudem erstellt sie jährlich eine Spendenstatistik.

Für die Gründung der Stiftung hat die Schweizerische Gemeinnützige Gesellschaft ein Startkapital zur Verfügung gestellt. Zudem haben zwanzig Kantone einen Beitrag an das Stiftungskapital gezahlt und damit ihre Solidarität mit der ZEWO bekundet. Der Stiftungsrat setzt sich aus Vertretern der gemeinnützigen Organisationen, der Politik, des Konsumentenschutzes, des Sozialbereichs

\footnotetext{
* Informationsdienst und Sammlungswesen, Stiftung ZEWO.
} 
sowie der Wirtschaft zusammen. Zurzeit haben drei Vertreter von Hilfswerken Einsitz im neunköpfigen Stiftungsrat.

In der Schweiz sind heute rund 450 gemeinnützige, Spenden sammelnde Organisationen berechtigt, das ZEWO-Gütesiegel zu führen. Diese lassen sich in einfache Organisationen, Mutterorganisationen und Unterorganisationen aufteilen. Von den rund 240 einfachen Organisationen und Mutterorganisationen sind knapp 65 Organisationen in der Entwicklungszusammenarbeit tätig.

\section{Kriterien der ZEWO zur Erteilung des Gütesiegels}

Die ZEWO zertifiziert Organisationen mit gemeinnütziger Zielsetzung, d.h. Hilfswerke, welche soziale, humanitäre oder soziokulturelle Aufgaben verfolgen oder sich dem Schutz der Um- und Mitwelt widmen.

$\mathrm{Zu}$ den wichtigsten Kriterien, die eine Organisation für den Erhalt des ZEWOSiegels erfüllen muss, gehören: funktionierende interne und externe Kontrollstrukturen, offene Information über Tätigkeit und Mittelverwendung, transparente Rechnungslegung sowie der wirtschaftliche, wirksame und zweckbestimmte Umgang mit den Spendengeldern. Die ZEWO überprüft, ergänzt und passt ihre Kriterien laufend den Entwicklungen an. Viele Kriterien der ZEWO spielen auch im Zusammenhang mit der Gestaltung der Organisation (Corporate Governance) eine bedeutende Rolle. Nachfolgend werden die wichtigsten Anforderungen der ZEWO kurz erläutert und der Bezug zur Corporate Governance aufgezeigt.

\section{Interne Kontrolle}

Die Grundfrage der internen Kontrolle umfasst die Aufgabenteilung und die Zusammenarbeit zwischen strategischer und operativer Ebene. Damit die Führungs- und Aufsichtskompetenzen des leitenden Organs kompetent wahrgenommen werden können, verlangt die ZEWO eine strikte Trennung zwischen dem leitenden und dem operativen Organ. Zudem schreibt die ZEWO vor, dass ein Mitglied des leitenden Organs nicht mit der als Geschäftsführerin oder Geschäftsführer amtierenden Person persönlich verbunden (verheiratet, verwandt, verschwägert, Lebensgemeinschaft) sein darf. Damit wird sichergestellt, dass eine wirksame Kontrolle des leitenden Organs über die Geschäftsführung möglich ist und keine Gefahr zur Verwässerung der Gewaltentrennung durch Personalunion oder Abhängigkeiten besteht. Zudem wird ermöglicht, dass das leitende Organ die Ziele der gemeinnützigen Organisation festlegt und über strategische Fragen entscheidet. Weiter wird durch die vorgeschriebene Trennung gewährleistet, dass das Management vom leitenden Organ gewählt und das Erreichen der Ziele kontrolliert werden kann. Eine Trennung zwischen leitendem und operativem Organ ermöglicht eine effektive Machtkontrolle.

Die ZEWO legt Wert darauf, dass die Entscheide des leitenden Organs breit abgestützt sind. Das leitende Organ muss aus diesem Grunde mindestens fünf Mitglieder umfassen, die nicht persönlich miteinander verbunden sein dürfen. Durch die kollektive Zeichnungsberechtigung wird weiter sichergestellt, dass die Vertretungsmacht nicht bei einer einzelnen Person liegt. 
Durch eine angemessene interne Kontrolle ist ein ordnungsgemässer Ablauf des betrieblichen Geschehens sicherzustellen. Bezüglich der Aufteilung der Kompetenzen zwischen der operativen Geschäftsleitung und dem leitenden Organ verlangt die ZEWO, dass diese in einem Geschäftsreglement klar geregelt werden. Dabei übernimmt die Organisation, d.h. der Vorstand bzw. der Stiftungsrat als leitendes Organ die Oberleitung und die Verantwortung dafür, dass das angestrebte Ziel in zweckmässiger, wirksamer und wirtschaftlicher Weise verfolgt wird.

Die ZEWO stellt mit diesen Kriterien die Gewaltentrennung zwischen dem leitenden und dem ausführenden Organ auch im Sinne der Corporate Governance sicher.

\section{Professionelles Arbeiten im Vorstand bzw. im Stiftungsrat}

Neben der strikten Trennung zwischen dem leitenden und dem ausführenden Organ ist wichtig, dass vor allem bei grossen Organisationen die Kontroll- und Aufsichtsfunktion durch einen ausreichend qualifizierten Vorstand bzw. Stiftungsrat wahrgenommen werden kann. Gerade bei grossen Organisationen fallen auch beim leitenden Organ umfangreiche Tätigkeiten an. Die Aufgaben sollten grundsätzlich ehrenamtlich wahrgenommen werden. Teilweise verlangen qualifizierte Stiftungsräte und Vorstandsmitglieder jedoch eine Entschädigung für die Erledigung der anspruchsvollen Arbeit. Die ZEWO hat ihre Bestimmung über die ehrenamtliche Tätigkeit des Vorstandes bzw. des Stiftungsrates deshalb angepasst und lässt neu auch Entschädigungen im leitenden Organ zu. Trotz der zulässigen Entschädigung des leitenden Organs muss dem gemeinnützigen Charakter der Organisation weiterhin dadurch Rechnung getragen werden, dass die Mitglieder des Stiftungsrates bzw. Vorstandes mindestens 100 Stunden ehrenamtlich leisten müssen. Für darüber hinausgehende zeitliche Belastungen können Entschädigungen ausgerichtet werden, deren Höhe den Anforderungen der Arbeiten angemessen sein soll.

\section{Finanzkontrolle}

Die Revisionsstelle übt im Zusammenhang mit der Corporate Governance eine Kontrollfunktion gegenüber dem leitenden Organ sowie der Geschäftsleitung aus und stellt sicher, dass die Mittel rechtmässig verwendet werden. Sie überprüft, ob Buchführung, Bilanz und Betriebsrechnung dem Gesetz und den Statuten entsprechen und ob das leitende Organ korrekt Rechenschaft ablegt.

Die ZEWO verlangt von allen Organisationen mit dem ZEWO-Gütesiegel, dass ihre Buchführung sowie die Jahresrechnung durch eine vom leitenden Organ und von der Geschäftsführung der Organisation unabhängige und fachlich befähigte Instanz geprüft werden. Je nach Grösse und Komplexität der Organisation werden hinsichtlich Befähigung der Revisionsstelle entsprechend hohe Anforderungen gestellt. Die Revision hat nach den Berufs- und Standesregeln der Treuhandkammer zu erfolgen. Die Revisionsstelle bestätigt in ihrem Bericht, dass sie unabhängig ist und keinerlei Weisungsgewalt untersteht. Ihre Prüfungsaufgaben umfassen die Frage, ob die Buchführung und die Jahresrechnung dem schweizerischen Gesetz, den Statuten sowie den einschlägigen ZEWO-Bestimmungen entsprechen. Mit dieser Vorschrift stellt die ZEWO 
sicher, dass das leitende Organ von Organisationen mit dem ZEWO-Gütesiegel über die Mittelverwendung korrekt Rechenschaft ablegt.

\section{Die Öffentlichkeit transparent informieren}

Transparenz der Organisationen gegenüber ihren Bezugsgruppen und Geldgebern ist für die ZEWO ein wichtiger Faktor. Die Thematik spielt auch in Zusammenhang mit der Corporate Governance eine zentrale Rolle. Insbesondere soll es den Bezugsgruppen einer gemeinnützigen, Spenden sammelnden Organisation möglich sein, in die relevanten Organisationsstrukturen zuverlässig Einblick zu nehmen, und die Organisationen sollen auch gehalten werden, angemessen zu informieren.

Das Gesetz sieht für Spenderinnen und Spender sowie Sponsoren gegenüber gemeinnützigen, Spenden sammelnden Organisationen kein Auskunftsrecht vor. Die ZEWO legt in diesem Fall in ihrem Sammlungsreglement fest, dass die Spenden sammelnden Organisationen den Spenderinnen und Spendern sowie den Mitgliedern und weiteren Bezugsgruppen auf Wunsch Informationen und Unterlagen zustellen müssen. Ein wichtiges Instrument zur Information der Öffentlichkeit sind ein aussagekräftiger Jahresbericht und eine detaillierte Jahresrechnung der jeweiligen Organisation.

\section{Lauterkeit und Ethik}

In der Kommunikation mit den Spenderinnen und Spendern sowie mit der Öffentlichkeit ist wichtig, dass sie den Anforderungen der Ethik und Lauterkeit genügt. Insbesondere müssen die Angaben in den Sammlungsaufrufen wie auch in den übrigen dem Sammlungszweck dienenden Publikationen wahrheitsgetreu und sachgemäss sein. Die freie Entscheidung zur Spende darf nicht durch emotionale, realitätsfremde Übertreibungen und inhaltliche Verzerrungen in den Sammlungsaufrufen beeinträchtigt werden. Dabei darf die Würde der Menschen, denen die Hilfe zugute kommen soll, nicht herabgesetzt werden. Weiter lehnt die ZEWO die Unterstützung von Einzelkinderpatenschaften sowie die Werbung mit solchen Patenschaften ab. Vielmehr soll die Unterstützung ganzer Projekte im Vordergrund stehen.

\section{Die neue Fachempfehlung in der Rechnungslegung (Swiss GAAP FER 21)}

Die neue Fachempfehlung in der Rechnungslegung, Swiss GAAP FER 21, soll die Jahresrechnungen der verschiedenen Hilfswerke standardisieren und dadurch die Transparenz erhöhen sowie die Vergleichbarkeit verbessern. Die Empfehlung trägt der Besonderheit der fehlenden Gewinnstrebigkeit sowie der Mittelbeschaffung von gemeinnützigen Organisationen Rechnung und ist für alle Organisationen mit dem ZEWO-Gütesiegel verbindlich. Grosse Organisationen müssen bereits im Berichterstattungsjahr 2004 nach FER 21 abschliessen, während kleine Organisationen ein Jahr länger Zeit haben.

Neu umfasst die Jahresrechnung sechs Bestandteile: Bilanz, Anhang, Mittelflussrechnung (für grosse Organisationen), Rechnung über die Veränderung des Kapitals sowie einen Leistungsbericht. Letzterer soll über die Wirtschaftlichkeit 
(Effizienz) und Leistungsfähigkeit (Effektivität) Auskunft geben und ergänzt als qualitatives Element den quantitativen Teil. Neu müssen zudem die erhaltenen Vorzugs- und Gratisleistungen wie zum Beispiel Freiwilligenarbeit, Sach- und Materialspenden und Sonderrabatte sowie andere Vergünstigungen (unentgeltliche Dienstleistungen bei Warenkäufen) offengelegt werden. Der Leistungsbericht unterliegt hingegen nicht der ordentlichen Prüfpflicht der Revisoren. Dieser wird nur von der ZEWO geprüft.

\section{Verwaltungskosten}

Spenderinnen und Spender, Sponsoren, institutionelle Geldgeber sowie die Medien verlangen immer öfter nach Kennzahlen zum Verwaltungsaufwand gemeinnütziger Organisationen. Die Zahlen sagen ihrer Meinung nach etwas über die Effizienz einer Organisation und über den sorgfältigen Umgang mit Spendengeldern aus. Die Situation ist komplex. Der wirtschaftliche Umgang einer gemeinnützigen Organisation mit ihren Spendengeldern lässt sich nicht auf Grund einer einzigen Zahl beurteilen. Zudem ist der Vergleich von Kennzahlen unterschiedlicher Organisationen problematisch.

Die neue Fachempfehlung in der Rechnungslegung (FER 21) legt zwar fest, welche Kosten offen zu legen sind, enthält aber keine Definition zur Zusammensetzung der Verwaltungskosten. Jede Organisation entscheidet weiterhin selbst, wie sich ihre Verwaltungskosten zusammensetzen. Heute weisen gemeinnützige Organisationen vor allem den allgemeinen Administrationsaufwand sowie den Sammlungsaufwand als Verwaltungsaufwand aus. Häufig nicht ausgewiesen werden die Projektbegleitungskosten. Je nachdem, wie viel Kosten eine Organisation auf die Projekte umlegt, variiert die Höhe der allgemeinen Verwaltungskosten.

Die ZEWO hat Mitte Juni 2004 ein Projekt zur Eruierung und Definition der Verwaltungskosten lanciert. Erste Auswertungen werden Ende 2005 erwartet.

\section{Wie bekannt ist das ZEWO-Label?}

Eine aktuelle Studie der Publitest AG zeigt, dass die spontane Bekanntheit des ZEWO-Gütesiegels in der Bevölkerung gegenüber 2001 mit 21 Prozent gleich geblieben ist. Bei den regelmässigen Spendern ist die spontane Bekanntheit im Jahr 2004 mit 43 Prozent deutlich höher als im Jahr 2001 (32\%). Die ZEWO ist in der Deutschschweiz bekannter als in der Westschweiz. Frauen, über 30jährige gut Verdienende (Einkommen über 6000 Franken) sowie Leute mit höherer Bildung sind mit dem ZEWO-Siegel ebenfalls besser vertraut als andere. Von allen Befragten finden 63 Prozent das Gütesiegel ganz oder teilweise glaubwürdig. Die Glaubwürdigkeit des Gütesiegels liegt bei Leuten, die gelegentlich oder regelmässig spenden, bei 73 Prozent.

\section{Schlussfolgerungen}

Die Förderung der Lauterkeit und Transparenz auf dem Spendenmarkt entspricht zweifellos einem Bedürfnis der Öffentlichkeit. Die ZEWO leistet dazu einen wichtigen Beitrag. Sie tut dies insbesondere, indem sie wirksame Kontrollstrukturen 
verlangt, eine Finanzkontrolle durch eine Revisionsstelle vorschreibt und sich für Transparenz und Offenlegung wichtiger Informationen in der Jahresrechnung sowie im Jahresbericht einsetzt. Gleichzeitig erhalten diese Kriterien auch im Zusammenhang mit der Corporate Governance immer mehr Bedeutung. Eine absolute Sicherheit im Umgang mit Spendengeldern kann auch von der ZEWO nicht gewährleistet werden. Sie soll aber eine sinnvolle Kontrolle über die Mittelverwendung von gemeinnützigen Organisationen sicherstellen.

Ziel der ZEWO ist es, auf der Basis einer konsolidierten Organisation den Dia$\log$ mit den gemeinnützigen Organisationen zu intensivieren und den Bekanntheitsgrad des Gütesiegels weiter zu steigern. Das ZEWO-Gütesiegel soll in einem breiten Bevölkerungskreis für einen vertrauenswürdigen Einsatz von Spendengeldern stehen. Zudem strebt die ZEWO mittelfristig eine bessere Verankerung in der Westschweiz an.

\section{Spendenstatistik}

Die ZEWO-Spendenstatistik umfasst für die Jahre 2001 und 2002 alle 300 Organisationen, die zu diesem Zeitpunkt das ZEWO-Gütesiegel führten. Die Auswertung beruht auf der schriftlichen Befragung und auf der Analyse der Jahresrechnungen. Die Erhebung der ZEWO basiert damit auf Angaben der Hilfswerke, während die Statistik des GfS-Spendenmonitors auf Befragungen von Spendern beruht.

Die Gesamteinnahmen der Organisationen mit dem ZEWO-Gütesiegel belaufen sich im Jahr 2002 auf 1,7 Milliarden Franken und sind damit gleich hoch wie im Vorjahr. Organisationen, die in der Entwicklungszusammenarbeit tätig sind, machen rund 40 Prozent des Gesamtertrags aus.

Von den gesamten Einnahmen bilden die privaten Spenden in Höhe von 610 Millionen Franken (36\%) sowie die Beiträge der öffentlichen Hand in Höhe von 580 Millionen Franken (34\%) die grössten Einnahmequellen der Spenden sammelnden Organisationen mit dem ZEWO-Gütesiegel. Die privaten Spendeneinnahmen gingen im Vergleich zum ausserordentlichen Spendenjahr 2001 um 13 Prozent zurück. Sie können unterteilt werden in Legate, Einzelspenden, institutionelle Spenden (Kirchen, Stiftungen, Vereine), Mitgliederbeiträge sowie andere Spendeneinnahmen. Von den privaten Spenden verzeichneten die Einzelspenden, die Mitgliederbeiträge und die institutionellen Spenden einen Rückgang. Am meisten gingen die Einzelspenden in Höhe von 100 Millionen Franken zurück. Dafür haben die Legate einen Zuwachs in Höhe von 15 Millionen Franken verzeichnet. Ebenfalls zugenommen haben die Beiträge der öffentlichen Hand um rund 40 Millionen Franken und die Eigenleistungen der Organisationen in Höhe von 10 Millionen Franken. 
Grafik 1: Verteilung der gesamten Einnahmen (1,7 Milliarden) der ZEW0-zertifizierten Organisationen ( $\mathrm{n}=\mathbf{2 8 0}), 2002$

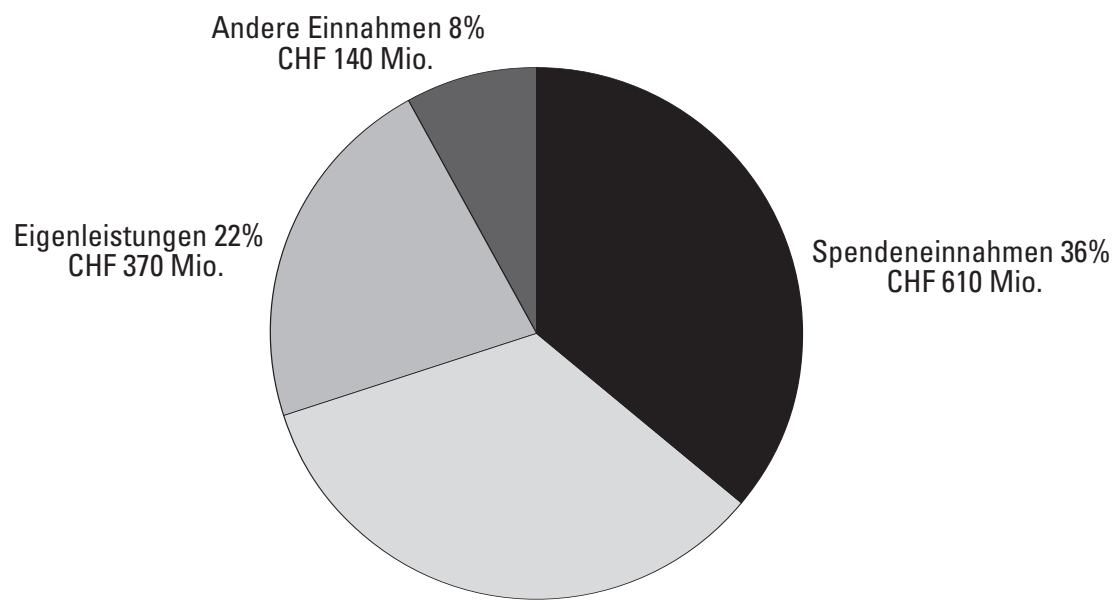

Beiträge der öffentlichen Hand $34 \%$ CHF 580 Mio.

Grafik 2: Verteilung der gesamten Einnahmen (1,7 Milliarden) der ZEWO-zertifizierten Organisationen $(\mathrm{n}=\mathbf{2 8 0})$, 2001

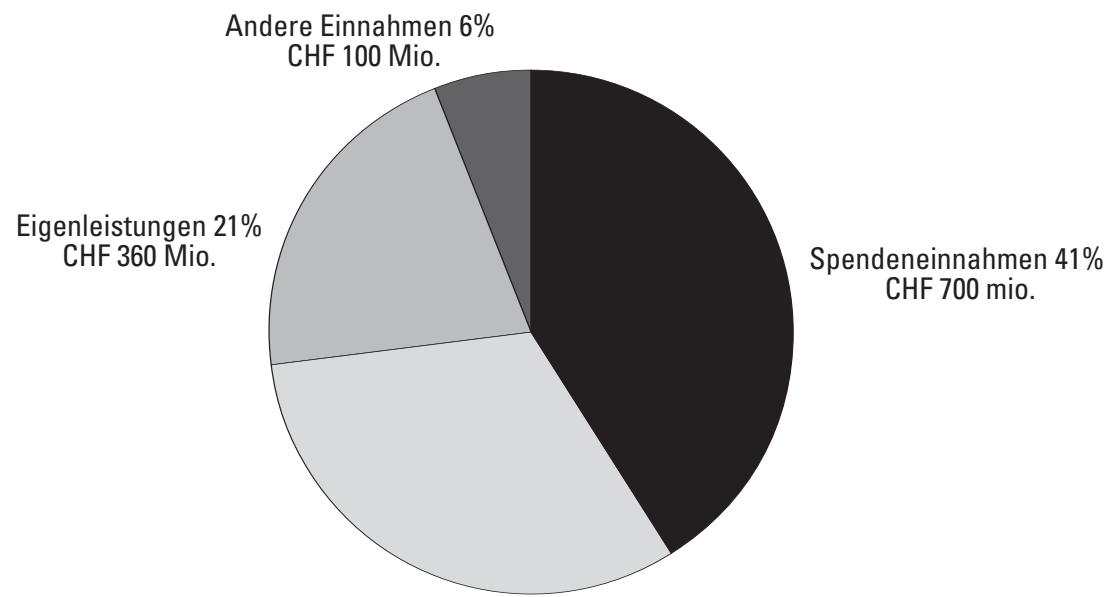

Beiträge der öffentlichen Hand $32 \%$ CHF 540 Mio. 
Grafik 3: Spendeneinnahmen (CHF 610 Millionen) der ZEWO-zertifizierten Organisationen ( $\mathrm{n}=\mathbf{2 8 0}), 2002$

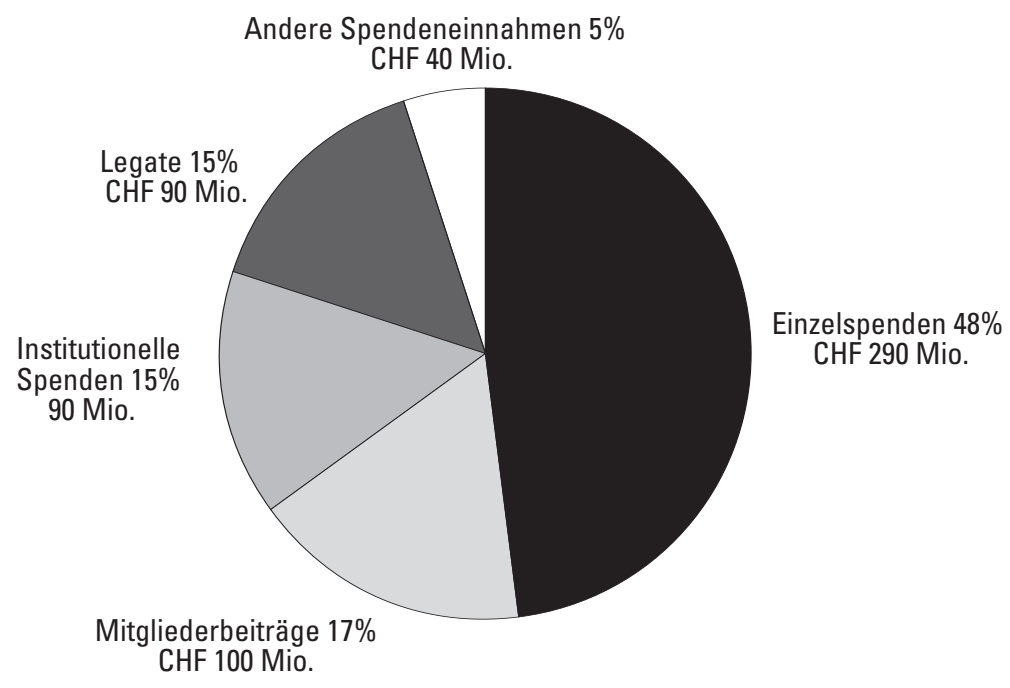

Grafik 4: Spendeneinnahmen (CHF 700 Millionen) der ZEWO-zertifizierten Organisationen $(\mathrm{n}=\mathbf{2 8 0}), 2001$

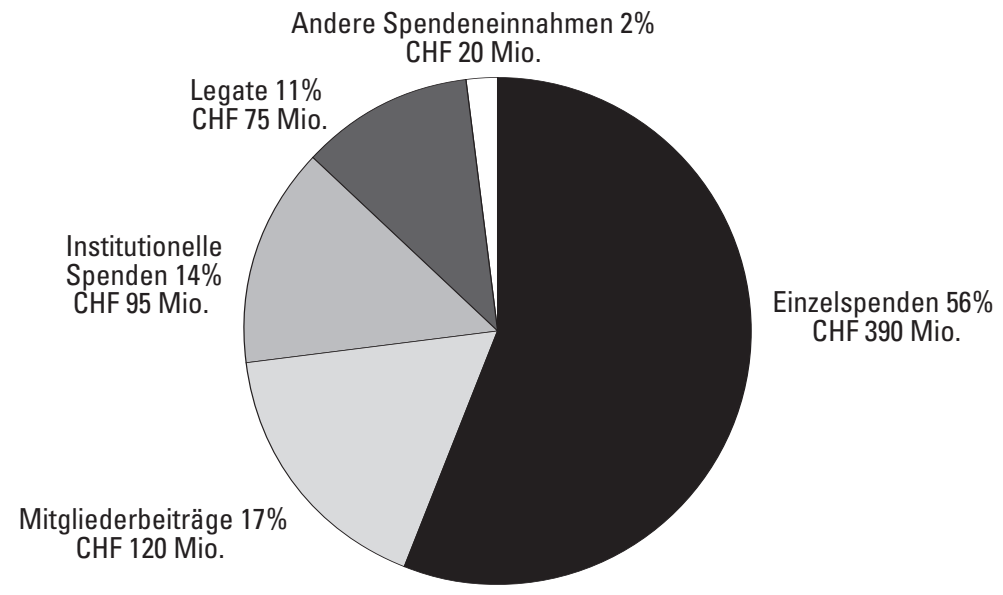

